\title{
SPIRAL STRUCTURE PARAMETERS IN THE SOLAR NEIGHBOURHOOD
}

\author{
D. FERNÁNDEZ, X. LURI, F. FIGUERAS AND J. TORRA \\ Dpt. d'Astronomia i Meteorologia, Universitat de Barcelona, \\ Av. Diagonal 647, E-08028 Barcelona, Spain
}

\begin{abstract}
.
Two samples of O- and B-type stars and Cepheids with Hipparcos data have been used to characterize galactic rotation and spiral arm kinematics in the solar neighbourhood. An extensive set of simulations has been performed in order to assess the capabilities of the method and its sensitivity to sample errors and biases in the adopted parameters.
\end{abstract}

\section{Introduction}

Young stars are good tracers of the galactic spiral structure in the solar neighbourhood, but, unfortunately, the observational data available up to now have not allowed to reach conclusive results on the parameters describing such structure. The accurate astrometric data provided by Hipparcos (ESA 1997) allow us to re-examine the kinematic behaviour of these stars and, even more, to evaluate the possibility to undertake this study using more sophisticated kinematic models.

In a previous paper (Torra et al. 2000) we characterized the structure and kinematics of the Gould Belt system, establishing its boundary to a distance of 600 pc from the Sun. Beyond this distance, the kinematic behaviour of young stars, in particular O-B stars and Cepheids, has to reflect the smooth galactic potential, that is the galactic rotation and the kinematics owing to the spiral structure. The study of the stellar velocity field outside the Gould Belt is undertaken in the present paper, considering both the current observational material - its errors and the sample's constraints - and, through realistic simulations, the robustness of the resolution process. In particular, for Cepheid stars, the influence of the period-luminosity relation (PLR) on the derived kinematic parameters is also evaluated. 


\section{The working samples}

\subsection{SAMPLE OF O- AND B-TYPE STARS}

The construction of this sample using Hipparcos data is fully described in Fernández (1998) and Torra et al. (2000). Due to the uncertainty on trigonometric parallax at large distances, these were computed following Crawford's (1978) photometric calibration. Preliminary results applying the LM method (Luri et al. 1996) to Hipparcos data indicate this calibration is good enough for our purposes (Jordi et al. 2000). Considering only those stars with an heliocentric distance $0.6<R<2 \mathrm{kpc}$, the sample with distances and proper motions contains 444 stars, whereas the subsample with available radial velocities (Grenier 1997) contains 304 stars.

\subsection{SAMPLE OF CEPHEID STARS}

The initial sample contains all the Hipparcos classical Cepheids. Astrometric data were taken from this catalogue and radial velocities from Pont

et al. (1994, 1997). Two PLR have been used for distance computation: Luri's (1999) relation, which corresponds to the short cosmic distance scale $\left(M_{v}=-1.08-2.72 \log P\right)$, and Feast \& Catchpole (1997) relation, which gives a long cosmic scale $\left(M_{v}=-1.41-2.81 \log P\right)$. In both cases, periods were obtained from Hipparcos and individual reddenings from Fernie et al.'s (1995) compilation (continuous updating). Up to $R=4 \mathrm{kpc}$, the sample with known distances and proper motions contains 203 stars and the subsample with known radial velocities contains 179 stars.

\section{Kinematic model}

Our kinematic model considers the systematic contributions of solar motion, differential galactic rotation (up to second-order approximation) and spiral arm kinematics (modeled in the frame of the Lin's theory). In addition to the solar motion, we derived the first- and second-order terms of the galactic rotation curve, the phase of the spiral structure at the Sun's position, the amplitudes in the perturbation of the velocity in the galactocentric and tangential directions and an adimensional parameter which takes into account the difference in the velocity dispersion between the solar-type stars and the considered stars. There are four free parameters: the number of spiral arms of the galaxy $(m)$, their pitch angle $(i)$, the galactocentric distance of the Sun $\left(\varpi_{\odot}\right)$ and the circular velocity at the Sun's position $\left(\Theta\left(\varpi_{\odot}\right)\right)$. A couple of values of these parameters were considered: $m=2$ or $4, i=-6^{\circ}$ or $-12^{\circ}, \varpi_{\odot}=7.1$ or $8.5 \mathrm{kpc}$ and $\Theta\left(\varpi_{\odot}\right)=184$ or $220 \mathrm{~km} \mathrm{~s}^{-1}$. The kinematic parameters were derived via weighted least squares fit, following an iterative scheme. Three cases were studied, solving 
the equations considering only radial velocities, only proper motions and the combined resolution (see Fernández et al. 2001).

\section{Test of robustness: simulations}

Numerical simulations were performed in order to quantitatively evaluate the biases in the kinematic model parameters induced by, among others, the observational errors, the incompleteness of the sample, the rejection of high residual stars and the correlations among the parameters. Both OB star and Cepheid samples were simulated considering the same spatial distribution than the real sample and a Schwarzschild distribution for the residual velocities. Solar motion, galactic rotation and spiral arm kinematics were introduced following our kinematic model. We assumed gaussian errors in distance, radial velocity and proper motions.

Detailed conclusions of these simulations can be found in Fernández et al. (2001). As main results, we conclude that the combined solution minimizes the biases induced by correlations. The observational errors in proper motion, radial velocities and distance produce biases less than 0.5 $\mathrm{km} \mathrm{s}^{-1}$ in the solar motion components and in the galactic rotation and spiral structure parameters. Due to the spatial distribution of the available data, the best distance intervals were found to be $0.6<R<2 \mathrm{kpc}$ for O-B stars and $R<4 \mathrm{kpc}$ for Cepheids.

\section{Results and discussion}

\subsection{GALACTIC ROTATION CURVE}

For the $A$ Oort constant, a discrepancy between the different solutions has been found: $A^{\mathrm{OB}} \approx 13.6 \pm 0.7 \mathrm{~km} \mathrm{~s}^{-1} \mathrm{kpc}^{-1}$ for O-B stars and $A_{\text {Luri }}^{\text {Cep }} \approx 16.7 \pm$ $0.6 \mathrm{~km} \mathrm{~s}^{-1} \mathrm{kpc}^{-1}, A_{\text {Feast }}^{\text {Cep }} \approx 15.0 \pm 0.6 \mathrm{~km} \mathrm{~s}^{-1} \mathrm{kpc}^{-1}$ for Cepheids (Luri's 1999 and Feast \& Catchpole's 1997 PLRs, respectively). This discrepancy has been extensively found in the literature (e.g. Hanson 1987, Metzger et al. 1997, Feast \& Whitelock 1997 and Mishurov \& Zenina 1999 obtained values between 11.3 and $18.8 \mathrm{~km} \mathrm{~s}^{-1} \mathrm{kpc}^{-1}$ ) and, as pointed out by Olling \& Merrifield (1998), it can be produced by the different spatial distribution of the samples. In agreement with Feast et al. (1998), we found a very small second-order term of the galactic rotation, compatible with a linear rotation curve in the solar neighbourhood.

\subsection{GALACTIC SPIRAL STRUCTURE}

Using both O-B and Cepheid stars data, we found an angular rotation velocity for the spiral pattern of $\Omega_{\mathrm{p}} \approx 30-35 \mathrm{~km} \mathrm{~s}^{-1} \mathrm{kpc}^{-1}$, incompatible with 
the classical value $13.5 \mathrm{~km} \mathrm{~s}^{-1} \mathrm{kpc}^{-1}$ proposed by Lin and collaborators in the later 60 s. Our value is in good agreement with the larger values recently published by Amaral \& Lépine (1997) and Mishurov et al. (1997, 1999). From this value we can conclude that the Sun is outside - though near - the corotation circle.

From the available data we are not able to decide on a galaxy with 2 or 4 spiral arms. Nowadays, this is an unsolved problem (see contradictory results in Amaral \& Lépine 1997, Mishurov \& Zenina 1999, Drimel 2000, Lépine et al. 2000). Nevertheless, good coherence is obtained to derive the phase of the spiral structure: from both samples, we obtained values in the rank $\left[330^{\circ}, 50^{\circ}\right]$, indicating the Sun is near the potential minimum of an arm. The values of the velocity amplitudes due to the spiral perturbation were found to be small, about $2 \mathrm{~km} \mathrm{~s}^{-1}$.

\section{Acknowledgements}

This work has been suported by the CICYT under contracts ESP97-1803 and ESP1999-1519-E.

\section{References}

Amaral, L.H., Lépine, J.R.D., 1997, MNRAS 286, 885

Crawford, D.L., 1978, AJ 83, 48

Drimel, R., 2000, A\&A 358, L13

ESA, 1997, The Hipparcos catalogue, ESA SP-1200

Feast, M.W., Catchpole, R.M., 1997, MNRAS 286, L1

Feast, M.W., Whitelock, P.A., 1997, MNRAS 291, 683

Feast, M.W., Pont, F., Whitelock, P.A., 1998, MNRAS 298, L43

Fernández, D., 1998, Degree of Physics (Master Thesis), Universitat de Barcelona, Spain (available in Spanish language from http://www.am.ub.es/ dfernand)

Fernández, D., Figueras, F., Torra, J., 2001, A\&A, in preparation (Kinematics of young stars. II. Galactic spiral structure)

Fernie, J.D., Beattie, B., Evans, N.R., Seager, S., 1995, IBVS 4148

Grenier, S., 1997, private communication

Hanson, R.B., 1987, AJ 94, 409

Jordi, C., Luri, X., Masana, E. et al., 2000, in: T. Lloy Evans (ed.), Procedings of the 24th meeting of the IAU, Highlights of Astronomy 12, in press

Lépine, J.R.D., Mishurov, Yu.N., Dedikov, S.Yu., 2000, astro-ph/0001216

Luri, X., 1999, private communication

Luri, X., Mennessier, M.O., Torra, J., Figueras, F., 1996, A\&AS 117, 405

Metzger, M.R., Caldwell, J.A.R., Schechter, P.L., 1997, AJ 115, 635

Mishurov, Yu. N., Zenina, I.A., Dambis, A.K., Mel'nik, A.M., Rastorguev, A.S., 1997, A\&A 323, 775

Mishurov, Yu. N., Zenina, I.A., 1999, A\&A 341, 81

Olling, R.P., Merrifield, M.R., 1998, MNRAS 297, 943

Pont, F., Mayor, M., Burki, G., 1994, A\&A 285, 415

Pont, F., Queloz, D., Bratschi, P., Mayor, M., 1997, A\&A 318, 416

Torra, J., Fernández, D., Figueras, F., 2000, A\&A 359, 82 